\section{One more imprinting review?}

\section{Genomic Imprinting: An Interdisciplinary Approach Edited by Rolf Ohlsson}

Springer-Verlag, \$156, ISBN 3-540-64667-1, 1999

\section{Reviewed by Frank Sleutels \& Denise P. Barlow}

Department of Molecular Genetics (H5),

The Netherlands Cancer Institute, Plesmanlaan 121, 1066CX Amsterdam, The Netherlands

Do you know how many review articles on genomic imprinting appeared during the last 12 months? Medline lists 46. In the midst of such largesse, a new collection of reviews needs something extra to stimulate a jaded appetite. In this case, the editor has for the most part chosen wisely, providing a collection that is informative and stimulating for those interested in epigenetics.

This book aims to provide an overview of genomic imprinting and of the epigenetic mechanisms that may regulate imprinting. The opening chapter, reviewing the kinship theory of imprinting, begins well-proposing a clear outline - but promptly throws this overboard, leaving the reader floundering in a sea of information. This chapter is difficult to read, and problematic for the experimental biologist with a strong urge to test predictions prior to publication. The text is enlivened by memorable metaphors, but the writing can lead to confusion-imprinted genes are described as "inactive in one sex"- when the authors presumably mean "inactive on one parental chromosome".

What a pleasure, then, to read the next three chapters, which are comprehensive, clear and provide useful basic information (including the latest molecular biological details). These three chapters cover aspects of imprinting that might earlier have been considered peripheral, but can now be viewed as models through which to explore general principles. The chapter on plants discusses how the link between imprinting in plants and animals is growing tighter, with the identification of the maternally expressed MEDEA gene, which inhibits embryonic growth. The chapter on insects is fascinating in its account of chromosome gymnastics, and excellent in its discussion of imprinting as applicable to diverse organisms. It makes the important statements that imprints are separate from the mechanism that uses them as cues, and the evolution of imprinted gene expression may be driven by other uniparental phenomena. The chapter on X inactivation offers a valuable perspective on this rapidly moving field by sum- marizing recent molecular data and comparing them with deductions drawn from earlier genetic analysis.

The next three chapters can be grouped together, as they cover imprinting in mammals. The chapter on mechanisms makes the point early on that imprinting should be regarded as a variation of existing epigenetic regulation, with no need to invoke a novel mechanism-the theme that provides the rationale for this book.

This chapter continues by detailing two alternative, though not mutually exclusive, models currently vying to explain imprinting: chromosomal subdomain signals residing in 'imprinting centres' may act as repressors in cis of large clusters of genes, or expressioncompetition may regulate a few closely linked genes. The authors discuss the pros and cons of these models, but given the large number of identified imprinted genes (as listed in the valuable appendix), the coverage is not complete. The principle data discussed are those from the Prader-Willi/Angelmann loci and the H19/Igf2 imprinted pair, which causes considerable overlap with the later chapter dealing with human disease. The chapter on cancer avoids this by focusing mainly on imprinted genes on human chromosome 11, particularly those involved in BeckwithWiedemann syndrome and Wilms tumour. Recent data are discussed, but the organization of the chapter makes understanding difficult. More diagrams would have helped, such as one clarifying how genetic and epigenetic mutations might affect expression.

The second part of the book, on possible 'players' in imprinting, reviews aspects of epigenetic gene regulation. Seven chapters cover transcription, epigenetics, Polycomb silencing, modifier genes, domains and boundaries, trans-sensing and nuclear architecture. The chapter on transcription stresses that positive and negative transcription factors can 'remodel' local chromatin structure, highlighting the involvement of cis-regulatory factors in eukaryotic gene regulation. But it neglects three key features of mammalian imprinted genes: all known imprinted genes have a CpG island-type promoter; a link between methylation and expression has already been shown; and expression of an imprinted gene can be regulated by an imprinted neighbour. These features are not unique to imprinted genes, but may provide clues to understanding their regulation.

The chapter on epigenetics remedies this somewhat by focusing on the function of DNA methylation in assembling the chromatin associated with silent genes. The significant experiments implicating DNA methylation are clearly outlined. But this chapter overlooks the finding that imprinted repression (in two known cases) does not depend directly on methylation of the repressed allele. In these cases, repression follows expression of an imprinted neighbouring non-coding gene, which appears to be the direct target of methylation-induced silencing. The earlier chapter on mechanisms stressed that imprinting should use existing epigenetic gene controls, but 'repression by a neighbour' may be the true novel feature of imprinting.

The chapter on Polycomb silencing presents a clear overview of chromatin mechanisms that preserve 'memory' of gene expression in clonal cell descendants. An apt parallel is drawn between the ability of Polycomb complexes to preserve cellular memory and the parental-specific memory seen in genomic imprinting. The chapters on domains and boundaries, trans-sensing and nuclear architecture provide clear overviews, and include sections linking these studies to possible imprinting mechanisms. The chapter on modifier genes includes a useful table of genetic modifier loci and a thoughtful appraisal of future directions for study.

How well, then, have the publishing team and editor achieved their goal? There are faults, but these lie mostly at the publishers' feet. Indexes at the start of each chapter would have helped, a glossary was needed for following texts overburdened with abbreviations and specialized names, the readability of some chapters needed improvement, and the overlaps between chapters should have been reduced. But this collection is a useful source of reference, and, at times, a quite stimulating read. A true advantage of a collection such as this is that you catch up on the reading you should have done and, much as supermarket convenience packages encourage increased consumption, you are motivated to read more. The words on the back of the book promising "an unrivaled indepth treatise ... aimed at any scientist irrespective of their field of study" are less wisely chosen than the contents, although on second thought, they compare well with the average standard of supermarket advertising. 